\title{
Atrial fibrillation and unresponsiveness with muscadol - A case report
}

Volume 6 Issue 4 - 2016

\section{Introduction}

Muscadol is a combination medication containing paracetamol $450 \mathrm{mg}$ and orphenadrine citrate $35 \mathrm{mg}$ used in the treatment of muscle spasms. The Paracetamol component is intended to provide analgesia, and the orphenadrine is an anticholinergic muscle relaxant. The adverse effects of paracetamol as well as orphenadrine individually are well described. Here we describe an uncommon adverse eventatrial fibrillation with unresponsiveness occurring in association with initiation of Muscadol treatment for osteoporosis.

\section{Case description}

An 89 year old woman with no previous comorbidities other than osteoporosis was brought to our ED by ambulance after she was found unresponsive for about 30 minutes at home. There was no witnessed seizures or abnormal movements. Her physical examination included: GCS 15 , T $37^{\circ} \mathrm{C}$, HR 130-140/minute, BP $120 / 80 \mathrm{mmHg}$, RR 16/minute, and pOx $99 \%$ on room air. Her cranial nerves were normal, pupils $6 \mathrm{~mm}$ and reactive, mucous membranes moist, bowel sounds present, and dry skin. Her 12-lead ECG demonstrated atrial fibrillation (which was a new finding compared to her old ECG). The patient was subsequently evaluated as a case of TIA secondary to atrial fibrillation. Her lab assays including complete blood count, electrolytes, renal function test were normal. Noncontrast brain CT revealed nothing indicating ischemia or hemorrhage. Upon reviewing her medication profile it was found that the patient was recently started on Muscadol (paracetamol $450 \mathrm{mg} /$ orphenadrine $35 \mathrm{mg}$ ) for osteoporosis 7days prior to the event. Consultations to cardiology and neurology service were obtained, and these included additional investigations of echocardiogram, the result of which was normal. At 4 hours after presentation, the patient was treated with Metoprolol $25 \mathrm{mg}$ orally and no further doses of muscadol were given. In $72 \mathrm{hrs}$ the patient reverted back to sinus rhythm, and she was discharged from the hospital.

\section{Discussion}

The physiologic mechanism for orphenadrine to cause atrial fibrillation is uncertain, but action as an anticholinergic agent is a possible explanation. A recent study of 484 people taking orphenadrine found atrial fibrillation in 12 of them $(2.48 \%)$. Of these $100 \%$ of them were female and $85.71 \%$ were $>60$ years. Although many conditions and medications can cause atrial fibrillation, Muscadol or any drug containing orphenadrine should be used with the understanding that atrial fibrillation is a recognized adverse event. In General drugs that increase or decrease adrenergic or vagal activity, such as sympathicomimetics, parasympathicomimetics, and their inhibitors, may be able to cause AF, especially in susceptible patients with a history of cardiovascular disease (disease is the substrate, drug is the trigger), but also in "healthy" patients. These drugs represent a substantial part of cardiovascular, respiratory, and central nervous system medications. ${ }^{1-5}$ Check Table for some of the examples

\section{Ayesha Parveen, Waleed Awad Salem, Abeer Kaled, Robert J Hoffman, Rawan Salameh \\ Department of Emergency Medicine, Hamad Medical Corporation, Qatar}

Correspondence: Ayesha Parveen, Department of Emergency Medicine, Hamad Medical Corporation, Doha, Qatar, Email ash_jamshed@yahoo.com

Received: September 20, 2016 | Published: September 26, 2016

\begin{tabular}{ll}
\hline Drug Class & Representative Drugs \\
\hline Anti-inflammatory drugs & NSAIDs \\
High dose & Methylprednisolone \\
Anti-convulsions drugs & Lacosemide \\
Dopamine agonist & Apomorphine \\
Cholinergic & Donepezil \\
Antipsychotics & Olanzapine \\
Antidepressants & Fluoxetine \\
Xanthine & Aminophylline \\
Miscellaneous & Nicotine \\
\hline
\end{tabular}

** Note: This list is not conclusive just examples.

\section{Conclusion}

The risk of orphenadrine-associated atrial fibrillation is greatest in elderly females above 60years of age. In our case, the atrial fibrillation was easily reversed by cessation of the drug and use of a beta blocker.

\section{Acknowledgments}

None.

\section{Conflicts of interest}

Author declares there are no conflicts of interest.

\section{Funding}

None.

\section{References}

1. Wolf PA, Abbott RD, Kannel WB. Atrial fibrillation as an independent risk factor for stroke: the Framingham study. Stroke. 1991;22(8):983-988.

2. Chugh SS, Blackshear JL, Shen WK, et al. Epidemiology and natural history of atrial fibrillation: clinical implications. J Am Coll Cardiol. 2001;37(2):371-378. 
3. Gupta AK, Maheshwari A, Tresch DD, et al. Cardiac arrhythmias in the elderly. Cardiac Electrophysiol Rev. 2002;6(1-2):120-128.

4. MacMahon JR. Letter: Atrial fibrillation and sympathomimetics. $J$ Pediatr. 1974; 84(4):613.
5. Van der Hooft CS, Heeringa J, van Herpen G, et al. Drug-Induced Atrial Fibrillation. J Am Coll Cardiol. 2004;44(11):2117-2124. 